\title{
How the axial anomaly controls flavor mixing among mesons
}

\author{
Francesco Giacosa, ${ }^{1,2, *}$ Adrian Koenigstein, ${ }^{2, \dagger}$ and Robert D. Pisarski ${ }^{3,+}$ \\ ${ }^{1}$ Institute of Physics, Jan Kochanowski University, ulica Swietokrzyska 15, 25-406 Kielce, Poland \\ ${ }^{2}$ Institute for Theoretical Physics, Johann Wolfgang Goethe University, Max-von-Laue-Straße 1, \\ 60438 Frankfurt am Main, Germany \\ ${ }^{3}$ Department of Physics, Brookhaven National Laboratory, Upton, New York 11973, USA
}

(Received 2 October 2017; published 22 May 2018)

\begin{abstract}
It is well known that, because of the axial anomaly in QCD, mesons with $J^{P}=0^{-}$are close to $S U(3)_{\mathrm{V}}$ eigenstates; the $\eta^{\prime}(958)$ meson is largely a singlet, and the $\eta$ meson an octet. In contrast, states with $J^{P}=1^{-}$ are flavor diagonal; e.g., the $\phi(1020)$ is almost pure $\bar{s} s$. Using effective Lagrangians, we show how this generalizes to states with higher spin, assuming that they can be classified according to the unbroken chiral symmetry of $G_{\mathrm{fl}}=S U(3)_{\mathrm{L}} \times S U(3)_{\mathrm{R}}$. We construct effective Lagrangians from terms invariant under $G_{\mathrm{fl}}$ and introduce the concept of hetero- and homochiral multiplets. Because of the axial anomaly, only terms invariant under the $Z(3)_{\mathrm{A}}$ subgroup of the axial $U(1)_{\mathrm{A}}$ enter. For heterochiral multiplets, which begin with that including the $\eta$ and $\eta^{\prime}(958)$, there are $Z(3)_{\mathrm{A}}$ invariant terms with low mass dimension which cause states to mix according to $S U(3)_{\mathrm{V}}$ flavor. For homochiral multiplets, which begin with that including the $\phi(1020)$, there are no $Z(3)_{\mathrm{A}}$ invariant terms with low mass dimension, and so states are diagonal in flavor. In this way, we predict the flavor mixing for the heterochiral multiplet with spin 1 as well as for hetero- and homochiral multiplets with spin 2 and spin 3.
\end{abstract}

DOI: 10.1103/PhysRevD.97.091901

\section{INTRODUCTION}

The theory of quarks and gluons, QCD, has two symmetries. There is an exact local symmetry of $S U(3)_{\mathrm{C}}$ color, which is responsible for the confinement of quarks and gluons into hadrons. For the light quark flavors of up, down, and strange quarks, $(u, d$, and $s)$, there is also an approximate chiral symmetry [1]. This is spontaneously broken in vacuum to a residual symmetry of $S U(3)_{\mathrm{V}}$ flavor. The up and down quarks are much lighter than hadronic scales, with the breaking of $S U(3)_{\mathrm{V}}$ flavor dominated by the effects of the strange quark, the mass of which is comparable to hadronic scales. Chiral symmetry is responsible for the most evident feature of hadrons, that, when it is spontaneously broken in vacuum, there is an octet of light pseudo-Goldstone bosons with spin parity $J^{P}=0^{-}$, which are pions, kaons, and the $\eta$ meson. Because of the mass of the strange quark, kaons, which include strange quarks, are heavier than pions.

This leaves a puzzle: why is there not a ninth light state, a $S U(3)_{\mathrm{V}}$ singlet? Instead, the corresponding state, the

\footnotetext{
fgiacosa@ujk.edu.pl

koenigstein@th.physik.uni-frankfurt.de

pisarski@bnl.gov
}

Published by the American Physical Society under the terms of the Creative Commons Attribution 4.0 International license. Further distribution of this work must maintain attribution to the author(s) and the published article's title, journal citation, and DOI. Funded by SCOAP. $\eta^{\prime}(958)$ meson, is unexpectedly heavy. Indeed, why should the pseudo-Goldstone bosons form states in representations of $S U(3)_{\mathrm{V}}$ flavor? Since the strange quark is so much heavier than the up and down quarks, why are the $\pi^{0}$ and $\eta$ not composed just of up and down quarks and the $\eta^{\prime}(958)$ purely strange [2]? The solution to these puzzles involves a global, axial symmetry of $U(1)_{\mathrm{A}}$, which, while valid classically, is reduced by quantum effects to a discrete subgroup of $Z(3)_{\mathrm{A}}$ [3]. This reduction in symmetry both pushes the mass of the $\eta^{\prime}(958)$ up and forces it to be close to a singlet under $S U(3)_{\mathrm{V}}$ flavor [2]. [In fact, the $\eta_{N}=$ $(\bar{u} u+\bar{d} d) / \sqrt{2}$ and $\eta_{S}=\bar{s} s$ do mix because of the strange quark mass.] This can be demonstrated in effective models, either nonlinear or linear [1-4].

What about other hadronic states? The lightest vector multiplet has $J^{P}=1^{-}$. This includes the $\rho(770)$ and $\omega(782)$ mesons, which are composed almost exclusively of up and down quarks, and the $\phi(1020)$ meson, which has mainly strange quarks. This difference can also be understood in effective models, such as through the WessZumino-Witten Lagrangian, in which effects due to the axial anomaly do not appear in mass terms, but only those of relatively high mass dimension.

This leads to the question which we address in this work: how does this striking difference in flavor mixing show up in hadronic multiplets with higher spin? We consider the case of the multiplet with $J^{P}=1^{+}$and multiplets with spin 2 and 3 . At the outset, we stress that our basic assumption is that we can classify the transformation properties of these 
multiplets according to the unbroken chiral symmetry. This is not self-evident for such heavy states, which experimentally have masses between 1 and $2 \mathrm{GeV}$. Nevertheless, this assumption allows us to make numerous predictions for their masses, flavor mixing, and decay modes. While most of the states which we discuss are not well measured experimentally, we hope that our comments might contribute to their further study.

In this work, we assume that the resonances under considerations are predominantly $\bar{q} q$ states (see Table I).
This is not trivial, since in principle each resonance is not a simple $\bar{q} q$ object but a superposition of various components, which include meson-meson bound states, tetraquarks, gluons, etc. Moreover, different approaches give rise to different interpretations of many resonances, e.g. Refs. [5-7]. For definiteness, we use the assignment presented in the "Quark Model" review of the Particle Data Group (PDG) [8]. While the nature of some resonances is disputed and certain parts of this assignment may change in the future, it gives us a valuable basis to start

TABLE I. Chiral multiplets, their currents, and transformations up to $J=3$. [ ${ }^{\star}$ and/or $f_{0}(1500)$; *^a mix of.] The first two columns correspond to the assignment suggested in the Quark Model review of the PDG [8], to which we refer for further details and references (see also the discussion in the text).

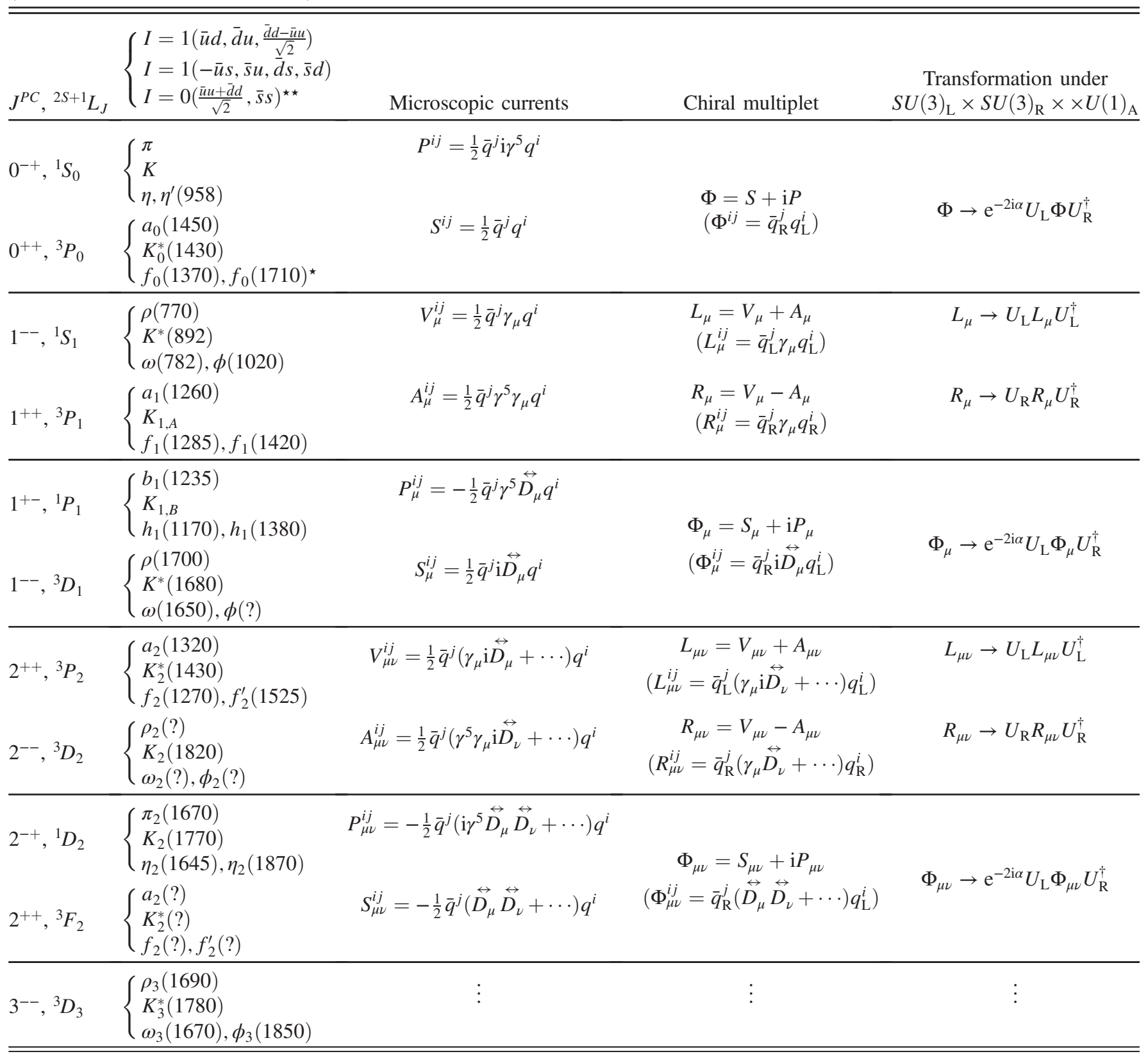


with. Moreover, our general considerations still apply, even if some of the specific assignments may change.

\section{CHIRAL SYMMETRY}

Suppressing color (indices), we denote quark fields as $q^{i}=(u, d, s)$. Left- and right-handed quarks fields are defined as

$$
q_{\mathrm{L}, \mathrm{R}}=\mathcal{P}_{\mathrm{L}, \mathrm{R}} q, \quad \bar{q}_{\mathrm{L}, \mathrm{R}}=\bar{q} \mathcal{P}_{\mathrm{R}, \mathrm{L}},
$$

where $\mathcal{P}_{\mathrm{L}, \mathrm{R}}=\left(\mathbb{1} \mp \gamma^{5}\right) / 2$ are orthogonal projectors, with $\mathcal{P}_{\mathrm{L}, \mathrm{R}}^{2}=\mathcal{P}_{\mathrm{L}, \mathrm{R}}$ and $\mathcal{P}_{\mathrm{L}} \mathcal{P}_{\mathrm{R}}=0$, taking $\left(\gamma^{5}\right)^{2}=\mathbb{1}$. Since $\bar{q}=q^{\dagger} \gamma^{0}$, in Eq. (1) the antiquark has a projector opposite to that of the quark. Under the global chiral symmetry of $G_{\mathrm{fl}} \times U(1)_{\mathrm{A}}=S U(3)_{\mathrm{L}} \times S U(3)_{\mathrm{R}} \times U(1)_{\mathrm{A}}$,

$$
q_{\mathrm{L}, \mathrm{R}} \rightarrow \mathrm{e}^{\mp \mathrm{i} \alpha / 2} U_{\mathrm{L}, \mathrm{R}} q_{\mathrm{L}, \mathrm{R}},
$$

where $U_{\mathrm{L}, \mathrm{R}}$ are rotations in $S U(3)_{\mathrm{L}, \mathrm{R}}$ and $\alpha$ is the parameter for an axial rotation in $U(1)_{\mathrm{A}}$. The flavor symmetry $G_{\mathrm{fl}}$ is exact in the limit that all quark masses vanish. It is spontaneously broken in the QCD vacuum to the diagonal subgroup of $S U(3)_{\mathrm{V}}$, under which $U_{\mathrm{L}}=U_{\mathrm{R}}=U_{\mathrm{V}}$. This generates an octet of massless Goldstone bosons.

The flavor symmetry is approximate once current quark masses are included. In QCD, the masses of the up, down, and strange quarks are $m_{u} \sim 5, m_{d} \sim 10$, and $m_{s} \sim 100 \mathrm{MeV}$, respectively. Symmetry breaking generates pseudo-Goldstone bosons, with the mass squared of the pion $m_{\pi}^{2} \propto\left(m_{u}+m_{d}\right)$ and that of the kaon $m_{K}^{2} \propto\left(m_{u}+m_{s}\right)$.

The axial symmetry is special. While the currents for $G_{\mathrm{fl}}$ are conserved in the limit of vanishing quark masses, that for the axial $U(1)_{\mathrm{A}}$ is proportional to the topological charge density $[3,9,10]$,

$$
\partial^{\mu}\left(\bar{q}^{i} \gamma_{\mu} \gamma_{5} q^{i}\right)=\frac{3 g^{2}}{16 \pi^{2}} \varepsilon^{\mu \nu \rho \sigma} \operatorname{tr}\left(G_{\mu \nu} G_{\rho \sigma}\right) .
$$

This reduces the $U(1)_{\mathrm{A}}$ symmetry to one of $Z(3)_{\mathrm{A}}$. This residual symmetry can be understood from the vertex generated by the zero modes of a single instanton, which for three flavors couples $u_{\mathrm{L}}, d_{\mathrm{L}}$, and $s_{\mathrm{L}}$ with $\bar{u}_{\mathrm{R}}, \bar{d}_{\mathrm{R}}$, and $\bar{s}_{\mathrm{R}}$. Under $q_{\mathrm{L}, \mathrm{R}} \rightarrow \mathrm{e}^{\mp \mathrm{i} \pi / 3} q_{\mathrm{L}, \mathrm{R}}$, this vertex changes as $\mathrm{e}^{2 \pi \mathrm{i}}$, and so is $Z(3)_{\mathrm{A}}$ invariant. The same applies to all multi-instanton interactions.

\section{HETEROCHIRAL SCALARS}

We begin by reviewing the effect of the axial anomaly on scalar fields. Because of confinement, we form fields which are color singlets (and so always implicitly sum over color indices) but transform nontrivially under flavor transformations. The simplest is to form a scalar by pairing a quark and an antiquark. Since the chiral projectors are orthogonal, pairing a quark and antiquark with the same chirality automatically vanishes, e.g., $\bar{q}_{\mathrm{L}} q_{\mathrm{L}}=\bar{q} \mathcal{P}_{\mathrm{R}} \mathcal{P}_{\mathrm{L}} q=0$. Instead, we must take

$$
\Phi^{i j} \equiv \bar{q}^{j} \mathcal{P}_{\mathrm{L}} q^{i}=\bar{q}_{\mathrm{R}}^{j} q_{\mathrm{L}}^{i},
$$

which under $G_{\mathrm{fl}} \times U(1)_{\mathrm{A}}$ transforms as

$$
\Phi \rightarrow \mathrm{e}^{-\mathrm{i} \alpha} U_{\mathrm{L}} \Phi U_{\mathrm{R}}^{\dagger} .
$$

Since we pair fields with opposite chirality, we term $\Phi$ as a heterochiral field. Consequently, the transformation of $\Phi$ under the flavor group involves both $U_{\mathrm{L}}$ and $U_{\mathrm{R}}$.

The components of $\Phi$ with $J^{P}=0^{-}$, given by $P=$ $\left(\Phi-\Phi^{\dagger}\right) / 2 \mathrm{i}$, are $\pi, K, \eta$, and $\eta^{\prime}(958)$; those with $J^{P}=0^{+}$, denoted as $S=\left(\Phi+\Phi^{\dagger}\right) / 2$, are assigned to $a_{0}(1450)$, $K_{0}^{*}(1430), f_{0}(1370)$, and $f_{0}(1710)$. In the quark model, their quantum numbers are ${ }^{2 S+1} L_{J}={ }^{1} S_{0}$ and ${ }^{3} P_{0}$, respectively.

Here, we assume that the $0^{+}$states below $1 \mathrm{GeV}$ are predominantly four-quark states [5-7,11-14], but this is secondary to our analysis. However, the nonstandard nature of the light scalar states is confirmed by various works, e.g. Refs. [5-7,11,12], where they emerge as dynamically generated states. The large- $N_{\mathrm{C}}$ behavior of the corresponding pole confirms this feature [15]. Yet, mixing is possible, and hence a certain $\bar{q} q$ amount into light scalars is present, but, as argued above, we regard it as subleading. While here we concentrate on predominantly $\bar{q} q$ states, the axial anomaly can play a role also for four-quark states [16]; this study is left for the future. Similarly, the above-listed scalar states above $1 \mathrm{GeV}$ are not simple $\bar{q} q$ resonances; mixing with four-quark components and with a scalar glueball is expected $[11,12,17-20]$. Due to all these complications, we do not attempt to study the anomalydriven mixing of the scalar-isoscalar $\bar{q} q$ components.

When spontaneous symmetry breaking occurs, we take the expectation value of $\Phi$ to be approximately diagonal in flavor, $\langle\Phi\rangle=\sqrt{3 / 2} \phi_{0} \mathbb{1}$, where $\phi_{0}=f_{\pi} \sim 93 \mathrm{MeV}$, which is a consequence of flavor symmetry.

In constructing an effective model of QCD at low energies, one includes all terms which are symmetric under $G_{\mathrm{fl}}[16,18,21-25]$. The simplest is to take traces of powers of $\Phi^{\dagger} \Phi$, such as $\operatorname{tr}\left(\Phi^{\dagger} \Phi\right), \operatorname{tr}\left(\Phi^{\dagger} \Phi\right)^{2}$, etc. These are all invariant under $U(1)_{\mathrm{A}}$. Terms which are only invariant under $Z(3)_{\mathrm{A}}$ begin with $\operatorname{det}(\Phi)$. This is generated by the zero modes of an instanton and so transforms as $\operatorname{det}(\Phi) \rightarrow$ $\mathrm{e}^{-3 \mathrm{i} \alpha} \operatorname{det}(\Phi)$ under $U(1)_{\mathrm{A}}$ [3]. The polynomial terms with the lowest mass dimension which are $Z(3)_{\mathrm{A}}$ invariant, Hermitian, and parity invariant are

$$
\begin{aligned}
\mathcal{L}_{\Phi}^{\text {anomaly }}= & -a_{\mathrm{A}}^{(1)}[\operatorname{det}(\Phi)+\text { c.c. }]-a_{\mathrm{A}}^{(2)}[\operatorname{det}(\Phi)+\text { c.c. }]^{2} \\
& -a_{\mathrm{A}}^{(3)}[\operatorname{det}(\Phi)-\text { c.c. }]^{2}-\cdots
\end{aligned}
$$

The first term is cubic in the fields and, when $\phi_{0} \neq 0$, drives the mass for the singlet pseudoscalar up, and that for the 
singlet scalar down $[3,16,23,24,26]$. The third term affects only isoscalar-pseudoscalar states, contributing to their mass squared as

$$
-\alpha_{\mathrm{A}} \eta_{0}^{2}=-\frac{\alpha_{\mathrm{A}}}{3}\left(\sqrt{2} \eta_{N}+\eta_{S}\right)^{2}
$$

where $\alpha_{\mathrm{A}} \sim a_{\mathrm{A}}^{(3)} \phi_{0}^{4}[18,22,25]$. It is in agreement with the Witten-Veneziano mass formula [27,28] and arises naturally when integrating out a pseudoscalar glueball [29]. The term $\sim a_{\mathrm{A}}^{(3)}$ contributes to the mixing angle between the $\eta_{N}$ and the $\eta_{S}$ as $2 \theta_{P}=-\arctan \left(2 \sqrt{2} \alpha_{\mathrm{A}} /\left(m_{K}^{2}-m_{\pi}^{2}-\alpha_{\mathrm{A}}\right)\right.$. For realistic values of $\alpha_{\mathrm{A}}$ [30], this explains the negative value of $\theta_{P} \sim-42^{\circ}$ observed experimentally [31]. The second term in Eq. (6) complicates the analysis. See also Refs. [4,31-33] for further phenomenological studies on the $\eta$ and $\eta^{\prime}(958)$.

\section{HOMOCHIRAL VECTORS}

Vector mesons are formed by pairing a quark and an antiquark with a Dirac matrix $\gamma_{\mu}$. Since $\gamma^{5}$ anticommutes with $\gamma_{\mu}$, we must pair a quark and antiquark with the same chirality

$$
L_{\mu}^{i j} \equiv \bar{q}_{\mathrm{L}}^{j} \gamma_{\mu} q_{\mathrm{L}}^{i}, \quad R_{\mu}^{i j} \equiv \bar{q}_{\mathrm{R}}^{j} \gamma_{\mu} q_{\mathrm{R}}^{i} .
$$

The left- and right-handed vector fields transform under the flavor group $G_{\mathrm{fl}} \times U(1)_{\mathrm{A}}$ as

$$
L_{\mu} \rightarrow U_{\mathrm{L}} L_{\mu} U_{\mathrm{L}}^{\dagger}, \quad R_{\mu} \rightarrow U_{\mathrm{R}} R_{\mu} U_{\mathrm{R}}^{\dagger} .
$$

We term these fields as homochiral, with $L_{\mu}^{i j}$ transforming as a two-index tensor under $S U(3)_{\mathrm{L}}$, and similarly for $R_{\mu}^{i j}$ under $S U(3)_{\mathrm{R}}$.

The vector fields themselves are automatically invariant under the axial $U(1)_{\mathrm{A}}$ symmetry, and so it is not possible to write a term which involves the axial anomaly using only these fields. Such effects are included by the coupling of the vector fields to the $\Phi$ 's [34], such as through Wess-ZuminoWitten type terms,

$$
\begin{gathered}
\varepsilon^{\mu \nu \alpha \beta} \operatorname{tr}\left[L_{\mu} \Phi\left(\partial_{\nu} \Phi^{\dagger}\right) \Phi\left(\partial_{\alpha} \Phi^{\dagger}\right) \Phi\left(\partial_{\beta} \Phi^{\dagger}\right)\right. \\
\left.\quad+R_{\mu} \Phi^{\dagger}\left(\partial_{\nu} \Phi\right) \Phi^{\dagger}\left(\partial_{\alpha} \Phi\right) \Phi^{\dagger}\left(\partial_{\beta} \Phi\right)\right] .
\end{gathered}
$$

Experimentally, the homochiral vector multiplet is well known. The vectors with negative parity, $J^{P}=1^{-}$, are given by $V_{\mu}=\left(L_{\mu}+R_{\mu}\right) / 2$; these are the $\rho(770), K^{*}(892)$, $\omega(782)$, and $\phi(1020)$. Axial vectors with positive parity, $J^{P}=1^{+}$, are $A_{\mu}=\left(L_{\mu}-R_{\mu}\right) / 2$ and comprise $a_{1}(1260)$, $K_{1, A}, f_{1}(1285)$, and $f_{1}(1420)$. In the quark model, their quantum numbers are ${ }^{2 S+1} L_{J}={ }^{3} S_{1}$ and ${ }^{3} P_{1}$, respectively. Note, the nature of axial-vector mesons is not yet clarified. As e.g. discussed in Refs. [35-37], $a_{1}(1260)$ can be described as a dynamically generated state rather than a standard $\bar{q} q$ state. For other phenomenological studies of (axial-)vector mesons (with emphasis on the mixing between the $K_{1, A}$ and the $K_{1, B}$ states), see Refs. [38-42].

There are three direct mass terms for the homochiral vector multiplets,

$$
\begin{aligned}
& m_{V}^{2} \operatorname{tr}\left(L_{\mu}^{2}+R_{\mu}^{2}\right)+\kappa \operatorname{tr}\left[m_{\mathrm{qk}}^{2}\left(L_{\mu}^{2}+R_{\mu}^{2}\right)\right] \\
& \quad+g_{1}^{2} \operatorname{tr}\left[\left(\Phi^{\dagger} L^{\mu}-R^{\mu} \Phi^{\dagger}\right)\left(L^{\mu} \Phi-\Phi R^{\mu}\right)\right] .
\end{aligned}
$$

The first term, $\sim m_{V}^{2}$, is invariant under the full flavor group of $G_{\mathrm{fl}}$; note that parity requires the masses of the left- and right-handed fields to be equal. The second term involves a diagonal matrix proportional to the current quark masses, $m_{\mathrm{qk}}=\operatorname{diag}\left(m_{\mathrm{u}}, m_{\mathrm{d}}, m_{\mathrm{s}}\right)$, and breaks the $S U(3)_{\mathrm{V}}$ symmetry for unequal quark masses. The third term generates the (standard) mass difference between chiral partners. For $\Phi \simeq \phi_{0}$, it reduces to $\phi_{0}^{2} \operatorname{tr}\left(A_{\mu}^{2}\right)$, and hence a mass term (proportional to $\phi_{0}$ ) is acquired by the axial-vector mesons only. Thus, the mass splitting among e.g. $\rho(770)$ and $a_{1}(1260)$ is generated by a nonvanishing condensate (just as for the splitting between the pion and $f_{N}$ ); see e.g. Refs. [43-45] for a detailed discussion.

Since these are homochiral fields, there is no term analogous to the $Z(3)_{\mathrm{A}}$ invariant terms for heterochiral fields, as in Eq. (6). Consequently, the mass eigenstates are naturally those of flavor and not of $S U(3)_{\mathrm{V}} \cdot \rho(770)$ and $\omega(782)$ are dominantly composed of up and down quarks, and the $\phi(1020)$, mainly strange; the associated mixing angle between the pure strange and nonstrange states is very small, $\theta_{V} \simeq-3.2^{\circ}$ [8]. The same seems to hold for axial-vector mesons. Although the nature of these states is still subject to ongoing debate [35-37,46], the states $f_{1}(1285)$ and $f_{1}(1420)$ appear to be predominantly nonstrange and strange, respectively [47]. Beyond these complications, which should be carefully addressed both theoretically and experimentally in the future, a small strange-nonstrange mixing of the underlying $\bar{q} q$ in the axial-vector channel is expected.

We now generalize this analysis to multiplets with higher spin. Assuming that we can classify states according to the unbroken chiral symmetry, we find that heterochiral states are mainly eigenstates of $S U(3)_{\mathrm{V}}$, and homochiral states, of flavor.

(In order to make the following argumentation as clear as possible, we provide Table I, where we list all chiral multiplets, their quark-antiquark currents, their transformation under $G_{\mathrm{fl}} \times U(1)_{\mathrm{A}}$, and their assignments to physical fields).

\section{HETEROCHIRAL VECTORS}

Instead of inserting a Dirac matrix between a quark and antiquark, we can use a gauge-covariant derivative, $D_{\mu}=\partial_{\mu}-\mathrm{i} g G_{\mu}$, 


$$
\Phi_{\mu}^{i j} \equiv \bar{q}_{\mathrm{R}}^{j}\left(\stackrel{\leftrightarrow}{D_{\mu}}+\cdots\right) q_{\mathrm{L}}^{i},
$$

where $G_{\mu}$ is the $S U(3)_{\mathrm{C}}$ gauge vector field (the gluon). As it is necessary to pair a left-handed quark with a right-handed antiquark, $\Phi_{\mu}$ is a heterochiral field, which transforms exactly like $\Phi$ in Eq. (5),

$$
\Phi_{\mu} \rightarrow \mathrm{e}^{-\mathrm{i} \alpha} U_{\mathrm{L}} \Phi_{\mu} U_{\mathrm{R}}^{\dagger} .
$$

Similarly, it is natural to form anomalous terms which are invariant under $G_{\mathrm{fl}}$ and $Z(3)_{\mathrm{A}}$ but not under $U(1)_{\mathrm{A}}$. Three terms analogous to Eq. (6) are

$$
\begin{aligned}
\mathcal{L}_{\Phi_{\mu}}^{\text {anomaly }}= & -b_{\mathrm{A}}^{(1)}\left[\operatorname{tr}\left(\Phi \times \Phi_{\mu} \cdot \Phi^{\mu}\right)+\text { c.c. }\right] \\
& -b_{\mathrm{A}}^{(2)}\left[\operatorname{tr}\left(\Phi \times \partial_{\mu} \Phi \cdot \Phi^{\mu}\right)+\text { c.c. }\right] \\
& -b_{\mathrm{A}}^{(3)}\left[\operatorname{tr}\left(\Phi \times \Phi \cdot \Phi_{\mu}\right)-\text { c.c. }\right]^{2}+\cdots
\end{aligned}
$$

plus other terms at sixth order, which we do not list here, and with the notation

$$
(A \times B)^{i i^{\prime}}=\frac{1}{3 !} \epsilon^{i j k} \epsilon^{i^{\prime} j^{\prime} k^{\prime}} A^{j j^{\prime}} B^{k k^{\prime}} .
$$

The vector multiplet decomposes into $\Phi_{\mu}=S_{\mu}+\mathrm{i} P_{\mu}$ [48]. The $P^{\mu}$ 's have $J^{P C}=1^{+-}$and may be the $b_{1}(1235)$, $K_{1, B}, h_{1}(1170)$, and $h_{1}(1380)$; in the quark model, they have ${ }^{2 S+1} L_{J}={ }^{1} P_{1}$. The $S^{\mu}$ 's, with $J^{P C}=1^{--}$, may be the $\rho(1700), K^{*}(1680), \omega(1650)$, and an excited $\phi(?)$ [49]; in the quark model, they have ${ }^{2 S+1} L_{J}={ }^{3} D_{1}$. Many of these states are controversial, e.g., the $h_{1}(1380)$ is not listed in Ref. [8].

Our predictions for these states are clear: if the first term in Eq. (14) dominates, both heterochiral vectors should be close to eigenstates of $S U(3)_{\mathrm{V}}$ and not diagonal in flavor. The second term contributes to decay channels for one $\Phi_{\mu}$ to two $\Phi$ 's. The third term only contributes to the $P_{\mu}$ states, $\sim-b_{\mathrm{A}}^{(3)} \phi_{0}^{4}\left(h_{1,0}^{\mu}\right)^{2}\left[h_{1,0}^{\mu}\right.$ is the singlet, a mixture of $h_{1}(1170)$ and $\left.h_{1}(1380)\right]$. The complete form of the effective Lagrangian will allow one to make detailed predictions for the mixing angles driven by the anomaly. As for the heterochiral scalars and tensors [see Eq. (23) below], we expect a non-negligible negative mixing angle for the heterochiral vectors.

\section{HOMOCHIRAL TENSORS}

Multiplets with spin 2 are formed by combining Dirac matrices and covariant derivatives. If we use one of each, we obtain left- and right-handed fields,

$$
\begin{aligned}
L_{\mu \nu}^{i j} & \equiv \bar{q}_{\mathrm{L}}^{j}\left(\gamma_{\mu} \stackrel{\leftrightarrow}{D_{\nu}}+\gamma_{\nu} \stackrel{\leftrightarrow}{D_{\mu}}+\cdots\right) q_{\mathrm{L}}^{i} \\
R_{\mu \nu}^{i j} & \equiv \bar{q}_{\mathrm{R}}^{j}\left(\gamma_{\mu} \stackrel{\leftrightarrow}{D_{\nu}}+\gamma_{\nu} \stackrel{\leftrightarrow}{D_{\mu}}+\cdots\right) q_{\mathrm{R}}^{i}
\end{aligned}
$$

These homochiral fields are invariant under $U(1)_{\mathrm{A}}$ and transform under $G_{\mathrm{fl}}$ as the homochiral vectors in Eq. (9),

$$
L_{\mu \nu} \rightarrow U_{\mathrm{L}} L_{\mu \nu} U_{\mathrm{L}}^{\dagger}, \quad R_{\mu \nu} \rightarrow U_{\mathrm{R}} R_{\mu \nu} U_{\mathrm{R}}^{\dagger} .
$$

As for the homochiral vector multiplet, the anomalous terms involve at least two derivatives.

The states $V_{\mu \nu}=\left(L_{\mu \nu}+R_{\mu \nu}\right) / 2$ with $J^{P C}=2^{++}$include the $a_{2}(1320), K_{2}^{*}(1430), f_{2}(1270)$, and $f_{2}^{\prime}(1525)$, with ${ }^{2 S+1} L_{J}={ }^{3} P_{2}$ in the quark model. There is also $A_{\mu \nu}$ with $J^{P C}=2^{--}$, with ${ }^{2 S+1} L_{J}={ }^{3} D_{2}$ in the quark model. These states are not well measured, except for the $K_{2}(1820)$. For an overview of tensor mesons, see Refs. [17,50-54].

Our analysis predicts that the effects of the anomaly are small and that these states are eigenstates of flavor. This agrees with experiment [8] and recent lattice studies [55], where the $f_{2}(1270)$ and $f_{2}^{\prime}(1525)$ correspond, to a very good approximation, to unmixed nonstrange and strange states, with a small mixing angle $\theta_{T} \simeq 3.2^{\circ}$ [8]. In general, masses and decays fit very nicely into the quark-nonet paradigm without any effect of the anomaly [56-58].

\section{HETEROCHIRAL TENSORS}

We cannot use two Dirac matrices to form spin-2 mesons, since the product of two Dirac matrices is $\gamma_{\mu} \gamma_{\nu}=g_{\mu \nu}-\mathrm{i} \sigma_{\mu \nu}$. The first term is a scalar and equivalent to previous fields. As $\sigma_{\mu \nu}$ is antisymmetric, this reduces to a vector field (for details, see Refs. [59,60]). We can also use two covariant derivatives,

$$
\Phi_{\mu \nu}^{i j} \equiv \bar{q}_{\mathrm{R}}^{j}\left(\stackrel{\leftrightarrow}{D_{\mu}} \stackrel{\leftrightarrow}{D_{\nu}}+\stackrel{\leftrightarrow}{D_{\nu}} \stackrel{\leftrightarrow}{D_{\mu}}+\cdots\right) q_{\mathrm{L}}^{i}
$$

To have spin 2, we must take the traceless part of $\Phi_{\mu \nu}$ [61]. This field is heterochiral and transforms like $\Phi$, with

$$
\Phi_{\mu \nu} \rightarrow \mathrm{e}^{-\mathrm{i} \alpha} U_{\mathrm{L}} \Phi_{\mu \nu} U_{\mathrm{R}}^{\dagger} .
$$

Terms which are invariant under $G_{\mathrm{fl}} \times Z(3)_{\mathrm{A}}$ but not under $U(1)_{\mathrm{A}}$ begin with

$$
\begin{aligned}
\mathcal{L}_{\Phi_{\mu \nu}}^{\text {anomaly }}= & -c_{\mathrm{A}}^{(1)}\left[\operatorname{tr}\left(\Phi \times \Phi_{\mu \nu} \cdot \Phi^{\mu \nu}\right)+\text { c.c. }\right] \\
& -c_{\mathrm{A}}^{(2)}\left[\operatorname{tr}\left(\partial_{\mu} \Phi \times \partial_{\nu} \Phi \cdot \Phi^{\mu \nu}\right)+\text { c.c. }\right] \\
& -c_{\mathrm{A}}^{(3)}\left[\operatorname{tr}\left(\Phi \times \Phi \cdot \Phi_{\mu \nu}\right)-\text { c.c. }\right]^{2}+\cdots
\end{aligned}
$$

in analogy with Eqs. (6) and (14).

The parity eigenstates are given by $\Phi_{\mu \nu}=S_{\mu \nu}+\mathrm{i} P_{\mu \nu}$. The odd parity states, $P_{\mu \nu}$, have $J^{P C}=2^{-+}$and ${ }^{2 S+1} L_{J}=$ ${ }^{1} D_{2}$ in the quark model. Candidates are the $\pi_{2}(1670)$, $K_{2}(1770), \eta_{2}(1645)$, and $\eta_{2}(1870)$ [62-64]. The even parity states, $S_{\mu \nu}$, have $J^{P C}=2^{++}$and ${ }^{2 S+1} L_{J}={ }^{3} F_{2}$ in the quark model. These are not well known experimentally and should be some sort of $a_{2}, K_{2}^{*}, f_{2}$, and $f_{2}^{\prime}$ states. 
As before, the first term contributes to masses for $\Phi_{\mu \nu}$ and will lead to the multiplet being close to eigenstates of $S U(3)_{\mathrm{V}}$. The second term contributes to decays of one $\Phi_{\mu \nu}$ to two $\Phi$ 's. The third term affects only pseudotensor mesons and delivers a contribution to the mass of the singlet state proportional to

$$
-\gamma_{\mathrm{A}}\left(\eta_{2,0, \mu \nu}\right)^{2}=-\frac{\gamma_{\mathrm{A}}}{3}\left(\sqrt{2} \eta_{2, N, \mu \nu}+\eta_{2, S, \mu \nu}\right)^{2}
$$

with $\gamma_{\mathrm{A}} \simeq c_{\mathrm{A}}^{(2)} \phi_{0}^{4}$. Indeed, a phenomenological study of known experimental widths and ratios [61] finds $\theta_{P T} \simeq-42^{\circ}$, and hence a surprisingly large mixing,

$$
\left(\begin{array}{l}
\eta_{2}(1645) \\
\eta_{2}(1870)
\end{array}\right)=\left(\begin{array}{cc}
\cos \theta_{P T} & \sin \theta_{P T} \\
-\sin \theta_{P T} & \cos \theta_{P T}
\end{array}\right)\left(\begin{array}{l}
\eta_{2, N} \\
\eta_{2, S}
\end{array}\right)
$$

where $\eta_{2, N}=(\bar{u} u+\bar{d} d) / \sqrt{2}$ and $\eta_{2, S}=\bar{s} s$. This result can be nicely explained by the presence of the axial anomaly in that sector. Moreover, considering only the third term in Eq. (20), the corresponding mixing angle $\theta_{P T}$ turns out to be negative (for realistic values of $\gamma_{\mathrm{A}}$ ) just as for heterochiral scalars,

$$
\theta_{P T} \simeq-\frac{1}{2} \arctan \left[\frac{2 \sqrt{2} \gamma_{\mathrm{A}}}{\left(m_{K_{2}(1770)}^{2}-m_{\pi_{2}(1670)}^{2}-\gamma_{\mathrm{A}}\right)}\right] .
$$

This result is approximate, since we neglect $c_{\mathrm{A}}^{(1)}$ and other mixing effects which are suppressed for a large number of colors. A more precise determination is possible with more experimental input.

\section{HOMOCHIRAL MESONS WITH $J=3$}

It is possible to extend our considerations to higher spin. As a further example, upon introducing the $J=3$ homochiral objects,

$$
\begin{aligned}
& L_{\mu \nu \rho}^{i j} \equiv \bar{q}_{\mathrm{L}}^{j}\left(\gamma_{\mu} D_{\nu} D_{\rho}+\cdots\right) q_{\mathrm{L}}^{i}, \\
& R_{\mu \nu \rho}^{i j} \equiv \bar{q}_{\mathrm{R}}^{j}\left(\gamma_{\mu} D_{\nu} D_{\rho}+\cdots\right) q_{\mathrm{R}}^{i},
\end{aligned}
$$

the nonet $V_{\mu \nu \rho}=\left(L_{\mu \nu \rho}+R_{\mu \nu \rho}\right) / 2$ corresponds to the rather well-known mesons with $J^{P}=3^{-}\left({ }^{2 S+1} L_{J}={ }^{3} D_{3}\right)$ including $\rho_{3}(1690), K_{3}^{*}(1780), \omega_{3}(1670)$, and $\phi_{3}(1850)$. Similar to the pseudotensor mesons, the chiral partners of the $3^{--}$ are not yet known. The strange-nonstrange mixing angle leading to $\omega_{3}(1670)$ and $\phi_{3}(1850)$ is listed by the PDG as $\sim 3^{\circ}$ [8], hence very small, in agreement with our expectations for homochiral multiplets.

\section{CONCLUSIONS}

Assuming that higher-spin states can be categorized according to the unbroken chiral symmetry, they can be classified according to whether they are homo- or heterochiral states. The principal prediction is whether the flavor mixing of strangeness follows $S U(3)_{\mathrm{V}}$ symmetry, for heterochiral multiplets, or just flavor, for homochiral multiplets.

Higher-spin states are usually classified using models of constituent quarks. Such a framework will automatically yield multiplets classified according to flavor. Thus homochiral multiplets should agree with the predictions of quark models.

In contrast, our analysis yields qualitatively new predictions for the heterochiral multiplets. There is evidence for this in the heterochiral tensor multiplet [61].

Besides masses and mixing, in the future, one can study also various decay channels by using Eqs. (14) and (20). Similar studies can be performed with other approaches, such as lattice gauge theory [55], chiral perturbation theory [17,53,54], Schwinger-Dyson equations, and so on. Our predictions can be tested experimentally at the ongoing effort at BESIII [65,66], as well as GlueX [67-69] and CLAS12 [70], both of which will start measurements soon, and later with PANDA at FAIR [71].

\section{ACKNOWLEDGMENTS}

The authors thank D. H. Rischke, Dennis D. Dietrich, the Chiral Field Theory Group, E. Oset, and C. Roberts for useful discussions. F. G. and R. D. P. thank the organizers of the 53rd Karpacz Winter School of Theoretical Physics, which engendered this Collaboration. R. D.P. thanks E. Oset and C. Roberts for discussions at NSTAR. The work of F. G. is supported by the Polish National Science Centre NCN through the OPUS Project No. 2015/ 17/B/ST2/01625. R. D. P. thanks the U.S. Department of Energy for support under Contract No. DE-SC0012704.
[1] A. W. Thomas and W. Weise, The Structure of the Nucleon (Wiley-VCH, Berlin, 2001), p. 389.

[2] D. J. Gross, S. B. Treiman, and F. Wilczek, Phys. Rev. D 19, 2188 (1979).

[3] G. 't Hooft, Phys. Rep. 142, 357 (1986).
[4] T. Feldmann, P. Kroll, and B. Stech, Phys. Rev. D 58, 114006 (1998).

[5] C. Amsler and F. E. Close, Phys. Lett. B 353, 385 (1995).

[6] E. Klempt and A. Zaitsev, Phys. Rep. 454, 1 (2007).

[7] J. R. Pelaez, Phys. Rep. 658, 1 (2016). 
[8] C. Patrignani et al. (Particle Data Group), Chin. Phys. C 40, 100001 (2016).

[9] S. L. Adler, Phys. Rev. 177, 2426 (1969).

[10] J. S. Bell and R. Jackiw, Nuovo Cimento A 60, 47 (1969).

[11] W. Ochs, J. Phys. G 40, 043001 (2013).

[12] S. Janowski, F. Giacosa, and D. H. Rischke, Phys. Rev. D 90, 114005 (2014).

[13] L. C. Gui, Y. Chen, G. Li, C. Liu, Y.-B. Liu, J.-P. Ma, Y.-B. Yang, and J.-B. Zhang (CLQCD Collaboration), Phys. Rev. Lett. 110, 021601 (2013).

[14] F. Brünner, D. Parganlija, and A. Rebhan, Phys. Rev. D 91, 106002 (2015); 93, 109903(E) (2016).

[15] J. R. Pelaez, Phys. Rev. Lett. 92, 102001 (2004).

[16] R. D. Pisarski and V. V. Skokov, Phys. Rev. D 94, 034015 (2016).

[17] L. S. Geng and E. Oset, Phys. Rev. D 79, 074009 (2009).

[18] A. H. Fariborz, R. Jora, and J. Schechter, Phys. Rev. D 72, 034001 (2005).

[19] M. Napsuciale and S. Rodriguez, Phys. Rev. D 70, 094043 (2004).

[20] F. Giacosa, Phys. Rev. D 80, 074028 (2009).

[21] S. Gasiorowicz and D. A. Geffen, Rev. Mod. Phys. 41, 531 (1969).

[22] C. Rosenzweig, J. Schechter, and C. G. Trahern, Phys. Rev. D 21, 3388 (1980).

[23] R. D. Pisarski, arXiv:hep-ph/9503330.

[24] D. Parganlija, F. Giacosa, and D. H. Rischke, Phys. Rev. D 82, 054024 (2010).

[25] D. Parganlija, P. Kovacs, G. Wolf, F. Giacosa, and D. H. Rischke, Phys. Rev. D 87, 014011 (2013).

[26] P. Kovcs, Z. Szp, and G. Wolf, Phys. Rev. D 93, 114014 (2016).

[27] G. Veneziano, Nucl. Phys. B159, 213 (1979).

[28] E. Witten, Ann. Phys. (N.Y.) 128, 363 (1980).

[29] W. I. Eshraim, S. Janowski, F. Giacosa, and D. H. Rischke, Phys. Rev. D 87, 054036 (2013).

[30] S. D. Bass and A. W. Thomas, Phys. Lett. B 634, 368 (2006).

[31] G. Amelino-Camelia et al., Eur. Phys. J. C 68, 619 (2010).

[32] R. Escribano and J. Nadal, J. High Energy Phys. 05 (2007) 006.

[33] B. Borasoy and R. Nissler, Eur. Phys. J. A 26, 383 (2005).

[34] H. Gomm, O. Kaymakcalan, and J. Schechter, Phys. Rev. D 30, 2345 (1984).

[35] S. Schael et al. (ALEPH Collaboration), Phys. Rep. 421, 191 (2005).

[36] M. Wagner and S. Leupold, Phys. Rev. D 78, 053001 (2008).

[37] S. Leupold and M. Wagner, Int. J. Mod. Phys. A 24, 229 (2009).

[38] Z. Q. Zhang, Z. W. Hou, Y. Yang, and J. Sun, Phys. Rev. D 90, 074023 (2014).

[39] R. Aaij et al. (LHCb Collaboration), J. High Energy Phys. 10 (2014) 064.

[40] X. Liu, Z. T. Zou, and Z. J. Xiao, Phys. Rev. D 90, 094019 (2014).
[41] H. Hatanaka and K. C. Yang, Phys. Rev. D 78, 074007 (2008).

[42] A. Ahmed, I. Ahmed, M. Ali Paracha, and A. Rehman, Phys. Rev. D 84, 033010 (2011).

[43] P. Ko and S. Rudaz, Phys. Rev. D 50, 6877 (1994).

[44] M. Urban, M. Buballa, and J. Wambach, Nucl. Phys. A697, 338 (2002).

[45] G. W. Carter, P. J. Ellis, and S. Rudaz, Nucl. Phys. A603, 367 (1996); A608, 514(E) (1996).

[46] L. Roca, E. Oset, and J. Singh, Phys. Rev. D 72, 014002 (2005).

[47] F. Divotgey, L. Olbrich, and F. Giacosa, Eur. Phys. J. A 49, 135 (2013).

[48] F. Giacosa, J. Sammet, and S. Janowski, Phys. Rev. D 95, 114004 (2017).

[49] M. Piotrowska, C. Reisinger, and F. Giacosa, Phys. Rev. D 96, 054033 (2017).

[50] W. Chen, Z. X. Cai, and S. L. Zhu, Nucl. Phys. B887, 201 (2014).

[51] V. V. Anisovich, Pis'ma Zh. Eksp. Teor. Fiz. 80, 845 (2004) [JETP Lett. 80, 715 (2004)].

[52] Z. G. Wang and Z. Y. Di, Eur. Phys. J. A 50, 143 (2014).

[53] R. Molina, D. Nicmorus, and E. Oset, Phys. Rev. D 78, 114018 (2008).

[54] D. Jido, J. A. Oller, E. Oset, A. Ramos, and U. G. Meissner, Nucl. Phys. A725, 181 (2003).

[55] R. A. Briceno, J. J. Dudek, R. G. Edwards, and D. J. Wilson, arXiv:1708.06667.

[56] L. Burakovsky and J. T. Goldman, Phys. Rev. D 57, 2879 (1998).

[57] V. Cirigliano, G. Ecker, H. Neufeld, and A. Pich, J. High Energy Phys. 06 (2003) 012.

[58] F. Giacosa, T. Gutsche, V. E. Lyubovitskij, and A. Faessler, Phys. Rev. D 72, 114021 (2005).

[59] S. Leupold, Phys. Rev. D 73, 085013 (2006).

[60] C. Terschlusen and S. Leupold, Phys. Lett. B 691, 191 (2010).

[61] A. Koenigstein and F. Giacosa, Eur. Phys. J. A 52, 356 (2016).

[62] B. Wang, C. Q. Pang, X. Liu, and T. Matsuki, Phys. Rev. D 91, 014025 (2015).

[63] B. Chen, K. W. Wei, and A. Zhang, Adv. High Energy Phys. 2013, 217858 (2013).

[64] A. V. Anisovich, C. J. Batty, D. V. Bugg, V. A. Nikonov, and A. V. Sarantsev, Eur. Phys. J. C 71, 1511 (2011).

[65] G. Mezzadri, Proc. Sci., EPS-HEP2015 (2015) 423.

[66] S. Marcello (BESIII Collaboration), J. Phys. Soc. Jpn. Conf. Proc. 10, 010009 (2016).

[67] H. Al Ghoul et al. (GlueX Collaboration), AIP Conf. Proc. 1735, 020001 (2016).

[68] B. Zihlmann (GlueX Collaboration), AIP Conf. Proc. 1257, 116 (2010).

[69] M. Shepherd, Proc. Sci., Bormio2014 (2014) 004.

[70] A. Rizzo (CLAS Collaboration), Proc. Sci., CD15 (2016) 060.

[71] M. F. M. Lutz et al. (PANDA Collaboration), arXiv:0903 .3905 . 